\title{
Holonomic Implementation of Three Wheels Omnidirectional Mobile Robot using DC Motors
}

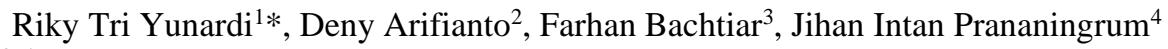 \\ 1, 2, 3,4 Department of Engineering, Faculty of Vocational, Universitas Airlangga, Indonesia \\ Email: *rikytriyunardi@vokasi.unair.ac.id
}

\begin{abstract}
In the Indonesian Wheeled Football Robot Contest (KRSBI) wheeled division, the robot that is made must be able to complete a predetermined task, one of which is the robot for chasing the ball and catching it. Holonomic is one of the methods used in navigating the omnidirectional movement of mobile robot applications. Because the movement is designed without changing the position of the robot in the direction of the facing, the omnidirectional wheels are used which has the ability to move freely in two directions. The mobile robot has three omnidirectional wheels and DC motors each used for movement. DC motors controlled by EMS 30A H-Bridge as a driver and Arduino Mega 2560 as the main microcontroller. Holonomic and inverse kinematic calculations are conducted to control the mobile robot movement of $x, y$, and $\omega$ toward angular velocity and direction of $\mathbf{s 1}, \mathrm{s} 2$, and $\mathrm{s} 3$ for each wheel. The length of the wheel axis to the middle of the body of robot is $160 \mathrm{~mm}$. In this study, a robot was implemented on the robot movement for moving forward, backward, sideways, and diagonal direction. Based on the data evaluation, it is determined that an angular error of $2.84 \%$ exists in the movement of the omnidirectional robot at a velocity of $0.256 \mathrm{~m} / \mathrm{s}$ to $1.403 \mathrm{~m} / \mathrm{s}$.
\end{abstract}

Keywords - Holonomic, three wheels omni directional, inverse kinematics, mobile robot, DC motor

\section{INTRODUCTION}

A mobile robot is designed to move through an environment and can determine its own motion path [1-3]. Mobile robots are divided into two groups, namely wheeled robots and legged robots, depending on the motion system [4]. But In division Football Wheeled Robot Contest Indonesia (KRSBI) must use a wheel that could be used more freely and be able to move on a flat surface. The robot must be able to perform programmed tasks, one of which is a robot to search and catch a ball.

The wheeled football robot division, robot should be able to play the ball in the field basically like a football player. The robot's limits, that is in its movement. The robot cannot accurately drive in the desired direction, the movement that is also done by the robot is the orientation and control of the movement of robot. The limitation of being able to move to the left, right, and diagonally without changing the orientation is also a problem. This causes the robot cannot play ball so well that a given mission cannot be completed properly [5-6].

One type of mobile robot that is the most widely used type of omni three-wheeled robot, known as omnidirectional mobile robot [7]. Omnidirectional has many advantages, such as flexibility in movement patterns, have the ability to move freely in both directions when compared with conventional two-wheeled robot as well as four-wheel drive [8-9]. The locomotion mechanism in the football robot uses forms of an omnidirectional wheel. With the same speed and acceleration the robot with omnidirectional wheels can drive in any direction. This would be very helpful given varying ball position on the robot. The omnidirectional design allowing it to be applied on the robot is three-wheel configuration, each wheel mounted 120 degrees differently. In this method the wheels are meant to be rotated either perpendicularly or parallel to the direction of motion [10].

The holonomic method in this study is applied in an omnidirectional mobile robot. It offers an omnidirectional three-wheel configuration to improve the mobility of the mobile robot to move with any orientation in either direction [11-12]. The three-wheel configuration provides the robot with omnidirectional mobility without needing to use a conventional driver system. Even the inverse kinematic model is introduced with an omni wheel drive which is modified to the robot dimensions. The DC motor is used for differential driving of the omni wheel. To get the velocity of each wheel, the inverse kinematics formula must first be obtained. A trajectory test is conducted to determine its ability to travel according to its direction of reference.

\section{HOLONOMIC OMNIDIRECTIONAL MOBILE ROBOT}

\section{A. Omnidirectional System of Motion}

Omnidirectional is defined as simply being able to move any direction. The occupied robotic space consists of three dimensions in mobile robots: the $\mathrm{x}$, $\mathrm{y}$ (point position on the robot) and the $\omega$ (robot orientation) [13]. Using omnidirectional motion method in geometry robotics as shown in Figure 1. The robot can move in any direction irrespective of position and orientation, so that the linear angular velocity $V x$ and $V y$ can be generated simultaneously. Regulation of these values can be achieved by using an omnidirectional motion method, such that the robot has three degrees of freedom (DOF) [14].

\section{B. Holonomic Motion System}

Holonomic motion system is a system that represents the number of degrees of freedom equal to the number of coordinates used to define system configuration [15-16]. Holonomic applied to a robot, without understanding the real form of mechanism. Mobile robots with a motion device that has three degrees of freedom in a field are a holonomic 
mobile robots. A holonomic motion system can help to plan the movement of the robot since there are no limits to the movement of the robot [17].

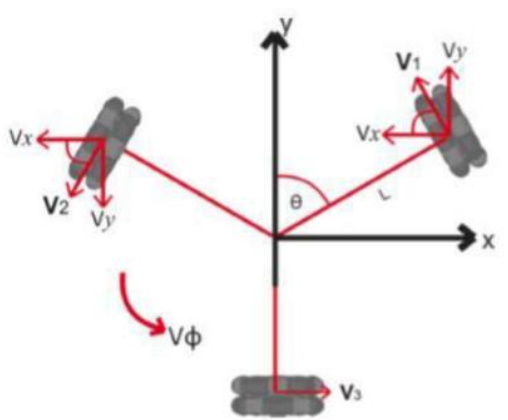

Fig. 1. The omnidirectional three-wheel configuration

The holonomic concept is applied to determine the angle where $\alpha$ is the motor axis angle drawn from the $\mathrm{x}$-axis coordinates of the robot frame. $\alpha$ is the motor axis angle from the $\mathrm{x}$ axis of the robot coordinate frame, each $\alpha 1=30^{\circ}, \alpha 2=$ $150^{\circ}$ and $\alpha 3=270^{\circ}$ [18]. The drive axis of the wheel $s$ as shown in Figure 2 , is $90^{\circ}$ or $\pi / 2$ of each $\alpha$. To solve the vector into its $\mathrm{x}$ and $\mathrm{y}$ components, we use some simple trigonometry.

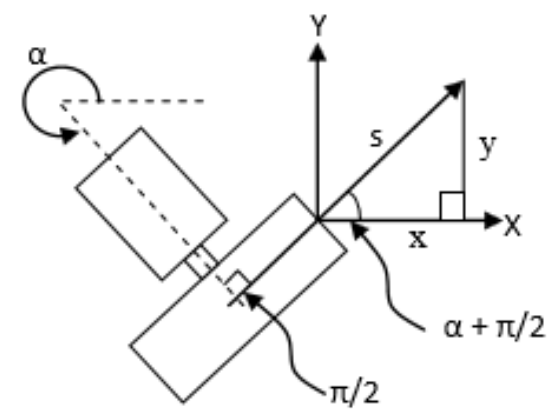

Fig. 2. The concept of wheel trigonometry

For each of the three wheels, the $\mathrm{x}$ and $\mathrm{y}$ components of the orientation of the robot are described in Equations (1) to (6).

$$
\begin{aligned}
& x_{1}=\cos \left(\alpha_{1}+\frac{\pi}{2}\right) s_{1} \\
& x_{2}=\cos \left(\alpha_{2}+\frac{\pi}{2}\right) s_{2} \\
& x_{3}=\cos \left(\alpha_{3}+\frac{\pi}{2}\right) s_{3} \\
& y_{1}=\sin \left(\alpha_{1}+\frac{\pi}{2}\right) s_{1} \\
& y_{2}=\sin \left(\alpha_{2}+\frac{\pi}{2}\right) s_{2} \\
& y_{3}=\sin \left(\alpha_{3}+\frac{\pi}{2}\right) s_{3}
\end{aligned}
$$

There is also a rotational component $\omega$, in which the robot can rotate its z-axis. Robot rotation is just a simple sum of each motor speed. Even if the motor turns in the opposite way, we generally still get the overall number of robot rotations. Just add the motor speed to find the robot rotation $\omega$. Motor speed for robot rotation $\omega$ in Equation (7).

$$
\omega=s_{1}+s_{2}+s_{3}
$$

\section{A. Inverse Kinematic}

Inverse kinematic for measuring wheel angular velocity. In this study the methods of inverse kinematics used to determine the DC motor speed [19-21]. The inverse kinematic formula for a robot with three wheels is defined as in Equation (8).

$$
\left(\begin{array}{l}
x \\
y \\
\omega
\end{array}\right)=\left(\begin{array}{ccc}
\cos \left(\alpha_{1}+\frac{\pi}{2}\right) & \cos \left(\alpha_{2}+\frac{\pi}{2}\right) & \cos \left(\alpha_{3}+\frac{\pi}{2}\right) \\
\sin \left(\alpha_{1}+\frac{\pi}{2}\right) & \sin \left(\alpha_{1}+\frac{\pi}{2}\right) & \sin \left(\alpha_{1}+\frac{\pi}{2}\right) \\
1 & 1 & 1
\end{array}\right)\left(\begin{array}{c}
s_{1} \\
s_{2} \\
s_{3}
\end{array}\right)
$$

Where $\mathrm{s} 1$ is motor velocity $1, \mathrm{~s} 2$ is motor velocity 2 and $\mathrm{s} 3$ is motor velocity 3 . Although $\mathrm{x}$ is the $\mathrm{x}$-axis, $\mathrm{y}$ is the $\mathrm{y}$-axis, and each robot rotation has a value of $1,-1$, and 0 .

By inverting Equation 1 means that get Equation (9).

$$
M^{-1}=\frac{1}{3}\left(\begin{array}{ccc}
-1 & \sqrt{3} & 1 \\
-1 & -\sqrt{3} & 1 \\
2 & 0 & 1
\end{array}\right)=\left(\begin{array}{ccc}
-0.33 & 0.58 & 0.33 \\
-0.33 & -0.58 & 0.33 \\
0.67 & 0 & 0.33
\end{array}\right)
$$

Then,

$$
\left(\begin{array}{l}
s_{1} \\
s_{2} \\
s_{3}
\end{array}\right)=\left(\begin{array}{ccc}
-0.33 & 0.58 & 0.33 \\
-0.33 & -0.58 & 0.33 \\
0.67 & 0 & 0.33
\end{array}\right)\left(\begin{array}{l}
x \\
y \\
\omega
\end{array}\right)
$$

\section{MATERIAL AND METHOD}

\section{A. Omni Directional Wheels}

Omni wheel is unusual in being able to travel independently in both directions [22]. This wheel usually turns like a circle and can move laterally around the outer diameter of the wheel using the screw. Omnidirectional wheels allow the robots to transform to holonomic robots from non-holonomic robots. A non-holonomic robot that uses regular wheels has only 2 operated DOF (Degree of Freedom), for example moving forward, backward, and rotating [23]. Holonomic omni wheels should overcome this issue since the wheels have 3 DOF. Unlike the nonholonomic robot, the holonomic omni directional robot is able to move in either direction without changing wheel orientation. Holonomic omni directional wheels move forward, backward, slide sideways, and rotate in a fixed position. Figure 3 shows the omni directional wheels. 


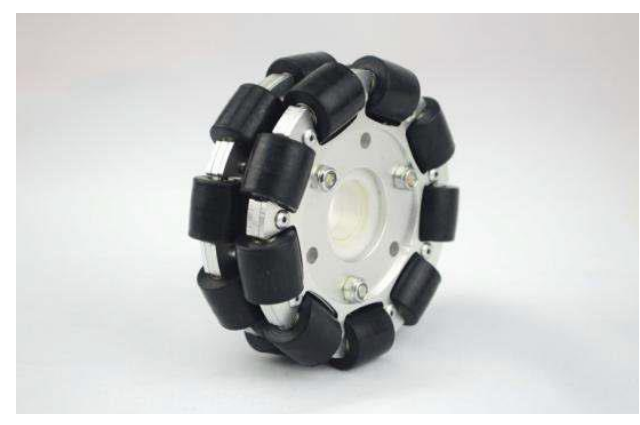

Fig. 3. The omni directional wheels

\section{B. $P G-45$ DC Motor}

DC motor is a motor that works with the input voltage in the form of DC voltage [24]. DC motors are widely used for designing a mobile robot. With a nominal voltage of 24 Volts, this PG-45 motor can produce a rotating speed of $3250 \mathrm{rpm}$. The power generated by this motor when given a 24 Volt voltage source is 22.5 Watts. The current generated in the motor is $0.2 \mathrm{~A}$.

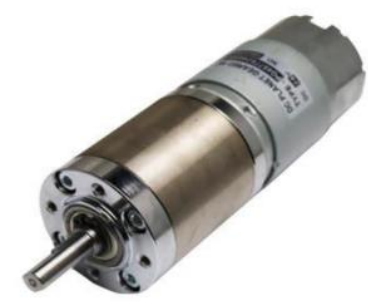

Fig. 4. PG-45 DC Motor

\section{EMS 30 A H-Bridge}

Embedded Module Series (EMS) 30 A H-Bridge is an $\mathrm{H}$ Bridge driver designed to produce a 2-way drive with a continuous current of up to $30 \mathrm{~A}$ at a voltage of 5.5 volts to
16 volts. This module is equipped with a load current sensor circuit that can be used as feedback to the controller.

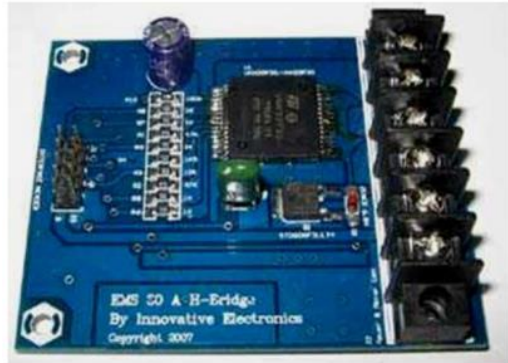

Fig. 5. EMS 30 A H-Bridge Module

\section{Arduino Mega 2560}

Arduino Mega 2560 is a microcontroller board based on Atmega 2560 [25-26]. Arduino Mega 2560 as shown in Figure 2.1 has 54 digital input / output pins, of which 15 pins can be used as PWM output, 16 pins as analog inputs, and 4 pins as UART (port serial hardware), $16 \mathrm{MHz}$ crystal oscillator, USB connection, power jack, ICSP header and reset button. This module is required to support the control system.

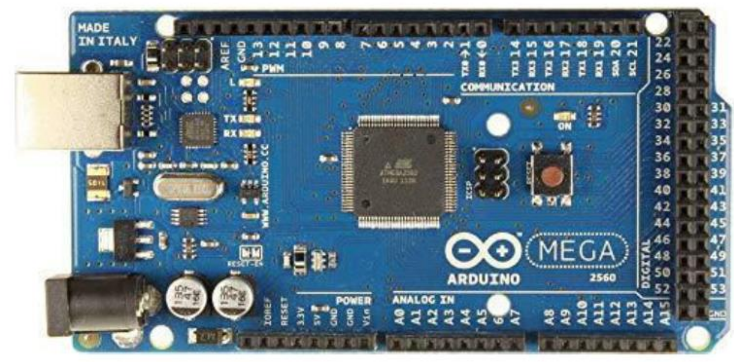

Fig. 6. Arduino Mega 2560

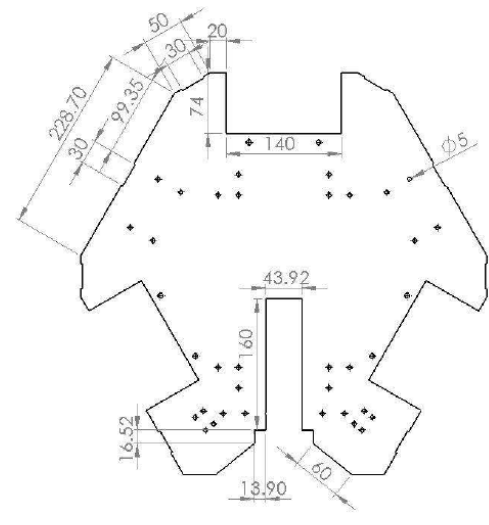

(a)

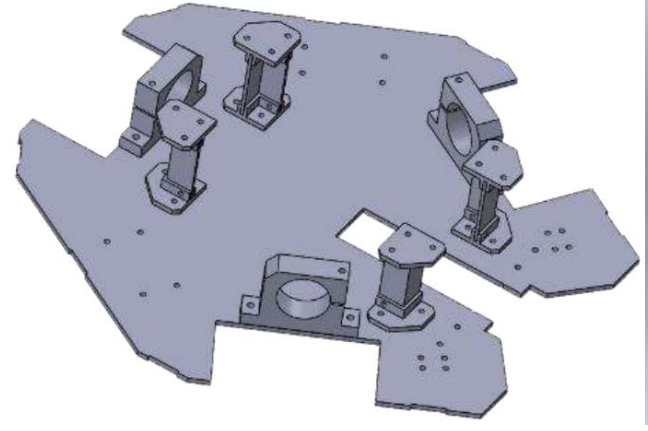

(b)

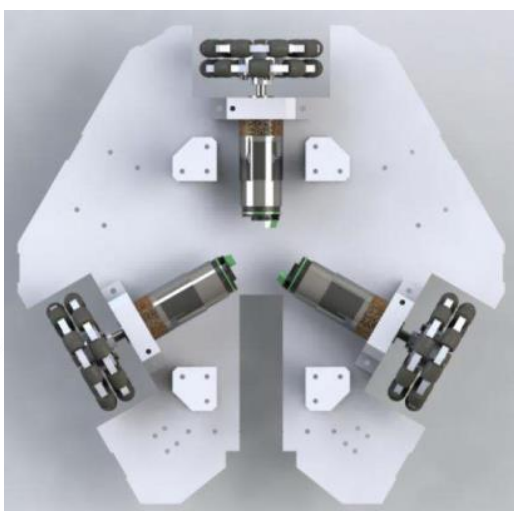

(c)

Fig. 7. (a) Chassis mechanical design; (b) chassis mechanical design perspective; (c) Omni wheel positioning design

\section{E. Inverse Kinematics of The Three-Joint Leg}

The chassis design has an area of $50 \mathrm{~cm} \times 51 \mathrm{~cm}$, as shown in Figure 7. The maximum weight of the robot that is allowed is $45 \mathrm{~kg}$, but the robot frame that is designed only weighs 10 $\mathrm{kg}$, so the material used for the frame is aluminum because it has a light and strong weight. The length of the wheel axis to the middle of the body of robot is $160 \mathrm{~mm}$. In addition, the robot is designed to have efficient movement and be able to control the ball well. The movements are agile and efficient as desired because in soccer games when fighting for the ball on the field. The robot mechanics design is made to be impact resistant, lightweight and not easily overturned when hit [2728]. This three omni directional robot movement system is triangular in shape with omni wheels placed at each end. 


\section{F. Movement Control System}

Movement control is used to monitor the PWM duty cycle, which acts to monitor each speed of motor to create vx, vy, and $\omega$ speed values [29-30]. The location of robot on the carpeted soccer field can be detected immediately at its turn.
The movement regulation block diagram can be seen in Figure 8. DC motor speed is obtained in the form of PWM and DC motor direction of motion which includes Clock Wise (CW) or Counter Clock Wise (CCW). Arduino Mega 2560, which gives the EMS 30 A-H Bridge a PWM value and drives a 45 DC motor.

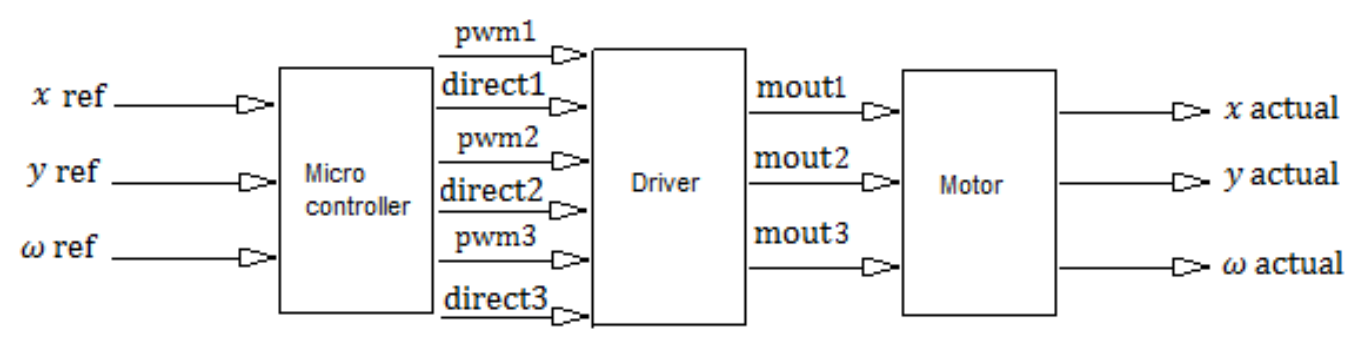

Fig. 8. Block diagram of the movement control

The test is carried out in a field measuring length and width 2 meters using a carpet floor that has been given a line according to the calculation in the formula, namely:
a. $360^{\circ}$ to move forward,
b. $45^{\circ}$ to move diagonally forward right,
c. $90^{\circ}$ to move right,
d. $135^{\circ}$ to move diagonally right backwards,
e. $180^{\circ}$ to move backwards,
f. $225^{\circ}$ to move diagonally left backward,
g. $270^{\circ}$ to move left,
h. $315^{\circ}$ to move diagonally forward left.

\section{RESULTS}

\section{A. Three Wheels Omnidirectional Mobile Robot}

A mobile robot with omnidirectional wheels shown in Figure 9 is suggested for the wheeled football robot division in this study. This robot comprises a wheel module composed of three omni directional wheels attached to each drive. The height of the ground platform is $70 \mathrm{~cm}$, and in this room between the platforms, additional components such as motor drives, controller, and battery are mounted.

\section{B. Direction Commands}

As instructions, more information on direction commands can be used in different ways, such as evaluating the speed of motor. Then s1, s2 and s3 must first be searched using the inverse kinematics formula as in Equation (11) until (7) to get the speed of each wheel.

$$
\left(\begin{array}{l}
s_{1} \\
s_{2} \\
s_{3}
\end{array}\right)=\left(\begin{array}{ccc}
-0.33 & 0.58 & 0.33 \\
-0.33 & -0.58 & 0.33 \\
0.67 & 0 & 0.33
\end{array}\right)\left(\begin{array}{l}
0 \\
1 \\
0
\end{array}\right)=\left(\begin{array}{c}
0.58 \\
-0.58 \\
0
\end{array}\right)
$$

For forward movement the values of s1, s2 and s3 are $(0.58$, $-0.58,0)$.

$$
\left(\begin{array}{l}
s_{1} \\
s_{2} \\
s_{3}
\end{array}\right)=\left(\begin{array}{ccc}
-0.33 & 0.58 & 0.33 \\
-0.33 & -0.58 & 0.33 \\
0.67 & 0 & 0.33
\end{array}\right)\left(\begin{array}{c}
0 \\
-1 \\
0
\end{array}\right)=\left(\begin{array}{c}
-0.58 \\
0.58 \\
0
\end{array}\right)
$$

For backwards movement the values of s1, s2 and s3 are ($0.58,0.58,0)$.

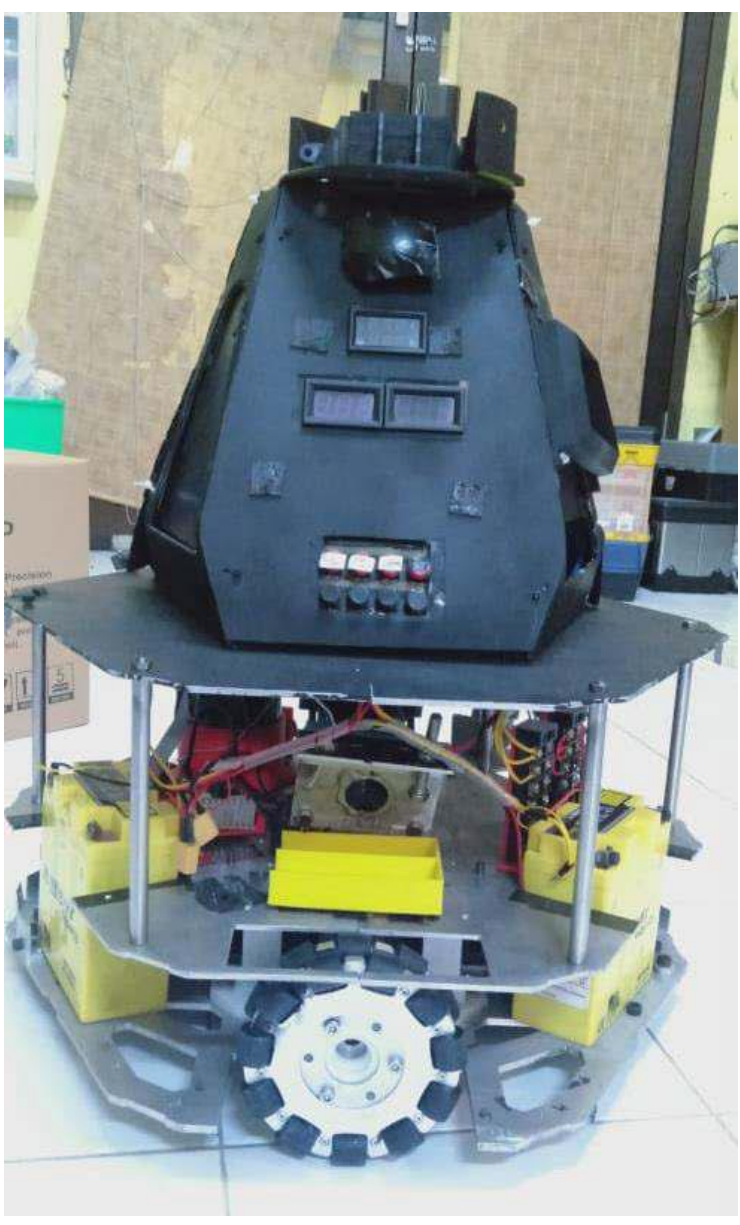

Fig. 9. The three wheels omnidirectional mobile robot

$$
\left(\begin{array}{l}
s_{1} \\
s_{2} \\
s_{3}
\end{array}\right)=\left(\begin{array}{ccc}
-0.33 & 0.58 & 0.33 \\
-0.33 & -0.58 & 0.33 \\
0.67 & 0 & 0.33
\end{array}\right)\left(\begin{array}{l}
1 \\
0 \\
0
\end{array}\right)=\left(\begin{array}{c}
-0.33 \\
-0.33 \\
0.67
\end{array}\right)
$$

For right movement the values of s1, s2 and s3 are (-0.33, $0.33,0.67)$.

$$
\left(\begin{array}{l}
s_{1} \\
s_{2} \\
s_{3}
\end{array}\right)=\left(\begin{array}{ccc}
-0.33 & 0.58 & 0.33 \\
-0.33 & -0.58 & 0.33 \\
0.67 & 0 & 0.33
\end{array}\right)\left(\begin{array}{c}
-1 \\
0 \\
0
\end{array}\right)=\left(\begin{array}{c}
0.33 \\
0.33 \\
-0.67
\end{array}\right)
$$

For left movement the values of s1, s2 and s3 are $(0.33,0.33$, -0.67). 


$$
\left(\begin{array}{l}
s_{1} \\
s_{2} \\
s_{3}
\end{array}\right)=\left(\begin{array}{ccc}
-0.33 & 0.58 & 0.33 \\
-0.33 & -0.58 & 0.33 \\
0.67 & 0 & 0.33
\end{array}\right)\left(\begin{array}{l}
1 \\
1 \\
0
\end{array}\right)=\left(\begin{array}{c}
0.25 \\
-0.91 \\
0.67
\end{array}\right)
$$

For diagonally forward right movement the values of s1, s2 and $\mathrm{s} 3$ are $(0.25,-0.91,0.67)$.

$$
\left(\begin{array}{l}
s_{1} \\
s_{2} \\
s_{3}
\end{array}\right)=\left(\begin{array}{ccc}
-0.33 & 0.58 & 0.33 \\
-0.33 & -0.58 & 0.33 \\
0.67 & 0 & 0.33
\end{array}\right)\left(\begin{array}{c}
1 \\
-1 \\
0
\end{array}\right)=\left(\begin{array}{c}
-0.91 \\
0.25 \\
0.67
\end{array}\right)
$$

For diagonally right backwards movement the values of $\mathrm{s} 1$, s2 and s3 are $(-0.91,0.25,0.67)$.

$$
\left(\begin{array}{l}
s_{1} \\
s_{2} \\
s_{3}
\end{array}\right)=\left(\begin{array}{ccc}
-0.33 & 0.58 & 0.33 \\
-0.33 & -0.58 & 0.33 \\
0.67 & 0 & 0.33
\end{array}\right)\left(\begin{array}{c}
-1 \\
1 \\
0
\end{array}\right)=\left(\begin{array}{c}
0.91 \\
-0.25 \\
-0.67
\end{array}\right)
$$

For diagonally forward left movement the values of s1, s2 and s3 are $(0.91,-0.25,-0.67)$.

$$
\left(\begin{array}{l}
s_{1} \\
s_{2} \\
s_{3}
\end{array}\right)=\left(\begin{array}{ccc}
-0.33 & 0.58 & 0.33 \\
-0.33 & -0.58 & 0.33 \\
0.67 & 0 & 0.33
\end{array}\right)\left(\begin{array}{c}
-1 \\
-1 \\
0
\end{array}\right)=\left(\begin{array}{c}
-0.25 \\
0.91 \\
-0.67
\end{array}\right)
$$

For diagonally left backwards movement the values of s1, s2 and $\mathrm{s} 3$ are $(-0.25,0.92,-0.67)$.

$$
\left(\begin{array}{l}
s_{1} \\
s_{2} \\
s_{3}
\end{array}\right)=\left(\begin{array}{ccc}
-0.33 & 0.58 & 0.33 \\
-0.33 & -0.58 & 0.33 \\
0.67 & 0 & 0.33
\end{array}\right)\left(\begin{array}{c}
0 \\
0 \\
-1
\end{array}\right)=\left(\begin{array}{l}
-0.33 \\
-0.33 \\
-0.33
\end{array}\right)
$$

For rotate left movement the values of s1, s2 and s3 are ($0.33,-0.33,-0.33)$.

$$
\left(\begin{array}{l}
s_{1} \\
s_{2} \\
s_{3}
\end{array}\right)=\left(\begin{array}{ccc}
-0.33 & 0.58 & 0.33 \\
-0.33 & -0.58 & 0.33 \\
0.67 & 0 & 0.33
\end{array}\right)\left(\begin{array}{c}
0 \\
0 \\
-1
\end{array}\right)=\left(\begin{array}{l}
0.33 \\
0.33 \\
0.33
\end{array}\right)
$$

For rotate right movement the values of s1, s2 and s3 are $(0.33,0.33,0.33)$.

\section{Tests The Forward Speed of Robot}

To evaluate the ratio between the forward velocity of the robot to the PWM input from the microcontroller, robot speed testing is performed. This measure is performed by giving motor driver the PWM input and adjusting the numerical value of the duty cycle. PWM varies from 30 to 255 such that it is possible to observe changes in the robot speed for a distance of 2 meters. Table 1 shows the PWM data and the forward speed of robot.

TABLE I. FORWARD SPEED OF THE ROBOT

\begin{tabular}{|c|c|c|c|}
\hline No & PWM & Time $(\boldsymbol{s})$ & Velocity $(\boldsymbol{m} / \mathbf{s})$ \\
\hline 1 & 255 & 1.425 & 1.403 \\
\hline 2 & 240 & 1.495 & 1.337 \\
\hline 3 & 230 & 1.545 & 1.294 \\
\hline 4 & 220 & 1.625 & 1.230 \\
\hline 5 & 210 & 1.665 & 1.201 \\
\hline 6 & 200 & 1.775 & 1.126 \\
\hline
\end{tabular}

\begin{tabular}{|c|c|c|c|}
\hline 7 & 190 & 1.845 & 1.084 \\
\hline 8 & 180 & 1.905 & 1.049 \\
\hline 9 & 170 & 2.025 & 0.987 \\
\hline 10 & 160 & 2.055 & 0.973 \\
\hline 11 & 150 & 2.205 & 0.907 \\
\hline 12 & 140 & 2.405 & 0.831 \\
\hline 13 & 130 & 2.505 & 0.798 \\
\hline 14 & 120 & 2.655 & 0.753 \\
\hline 15 & 110 & 2.805 & 0.713 \\
\hline 16 & 100 & 2.885 & 0.693 \\
\hline 17 & 90 & 3.025 & 0.661 \\
\hline 18 & 80 & 3.355 & 0.596 \\
\hline 19 & 70 & 3.505 & 0.570 \\
\hline 20 & 60 & 3.655 & 0.547 \\
\hline 21 & 50 & 4.123 & 0.485 \\
\hline 22 & 40 & 4.655 & 0.429 \\
\hline 23 & 30 & 7.805 & 0.256 \\
\hline & & & \\
\hline
\end{tabular}

\section{Tests The Angle of Rotation of Robot}

The rotary angle of the robot is measured to determine the orientation of the face of robot as it rotates. This test is conducted by giving the robot commands to rotate at a given angle. Then observed at the angle resulting from the movement of robot to the real angle change. Table 2 shows the orientation angle of rotation of robot.

TABLE II. ORIENTATION ANGLE OF ROTATION OF ROBOT

\begin{tabular}{|c|c|c|c|}
\hline No & Real Angle & Angle Result & Error (\%) \\
\hline 1 & $45^{\circ}$ & $51.2^{\circ}$ & 12.1 \\
\hline 2 & $90^{\circ}$ & $88.8^{\circ}$ & 1.35 \\
\hline 3 & $135^{\circ}$ & $140.5^{\circ}$ & 3.91 \\
\hline 4 & $180^{\circ}$ & $178.7^{\circ}$ & 0.73 \\
\hline 5 & $225^{\circ}$ & $223.2^{\circ}$ & 0.8 \\
\hline 6 & $270^{\circ}$ & $275.6^{\circ}$ & 2.03 \\
\hline 7 & $315^{\circ}$ & $312^{\circ}$ & 0.96 \\
\hline 8 & $360^{\circ}$ & $3.2^{\circ}$ & 0.88 \\
\hline
\end{tabular}

From these data it can be determined that the average angle orientation error to which the robot is moving in the direction defined is $2.84^{\circ}$.

\section{E. Tests the movement of Robot Based on The Trajectory}

The object of this test is to determine the movement of the robot towards the actual trajectory and compare it to direct observation results in the field. This test is carried out by providing a route connecting the point of departure to the point of destination like forms a line. System tested at constant speed. Trajectory data are observed and compared at the actual coordinate with the target coordinates of each trajectory. As shown in Figure 10 to Figure 14, the results of each test are shown in the form of a comparison graph between the target coordinates and the trajectory produced during the test. 


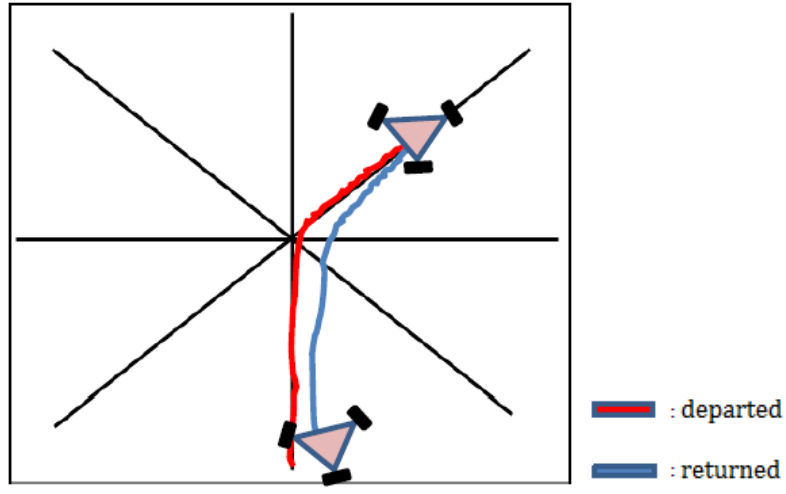

Fig. 10. The trajectory 1

Figure 10 is shown a trajectory 1 , the results of observations with the movement trajectory is forward, diagonally forward right, left diagonal backward, reverse, and the last step is stop.

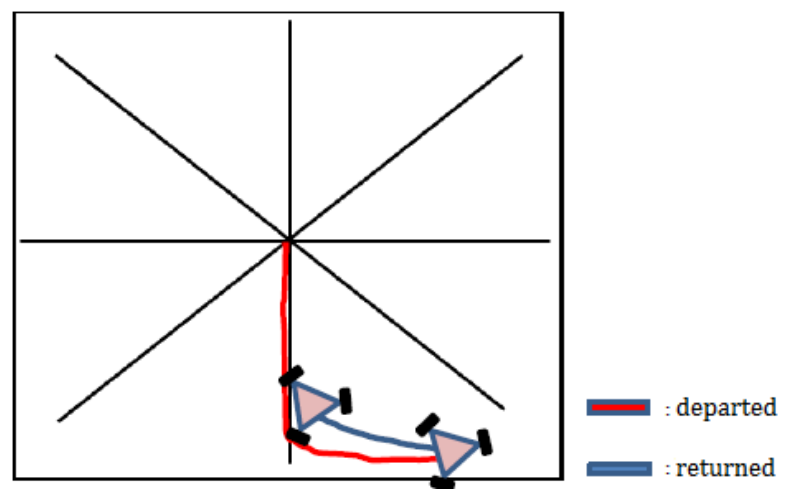

Fig. 11. The trajectory 2

In Figure 11 shown for illustration of the trajectory robot move backward, swipe right, then swipe left and forward, then stop.

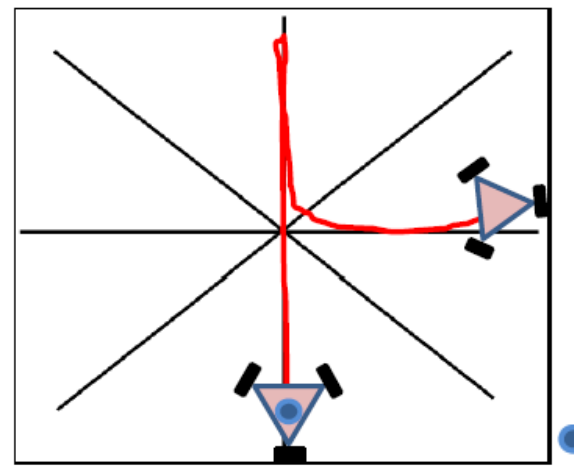

: departed point

Fig. 12. The trajectory 3

The trajectory movements used in Figure 12 are forward, backward, right shift and stop.

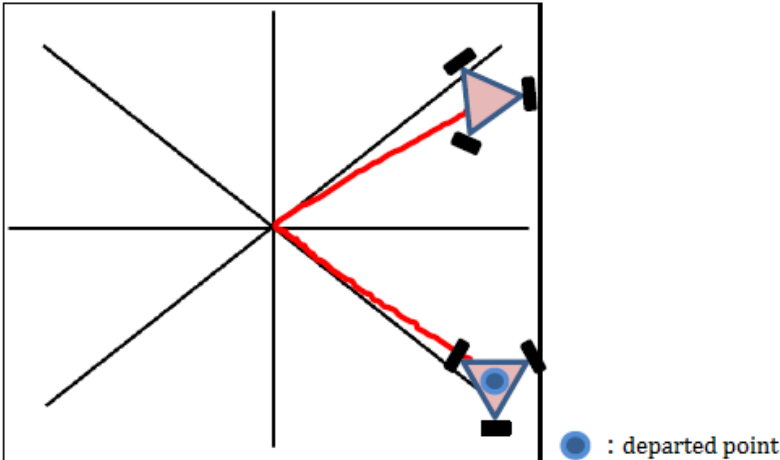

Fig. 13. The trajectory 4

From Figure 13 and Figure 13, it can be seen that the movement of the robot is made just departing. But in both images, the robot is only varied with two movements. Figure 13 is a movement to the left diagonally forward and diagonally forward right, while Figure 14 is a forward and slide right then stop. In trajectory 4 , the robot changes its orientation and stops not at the reference line. While in Trajectory 5 robot stops with the same orientation, but the midpoint of the robot is not in the reference line.

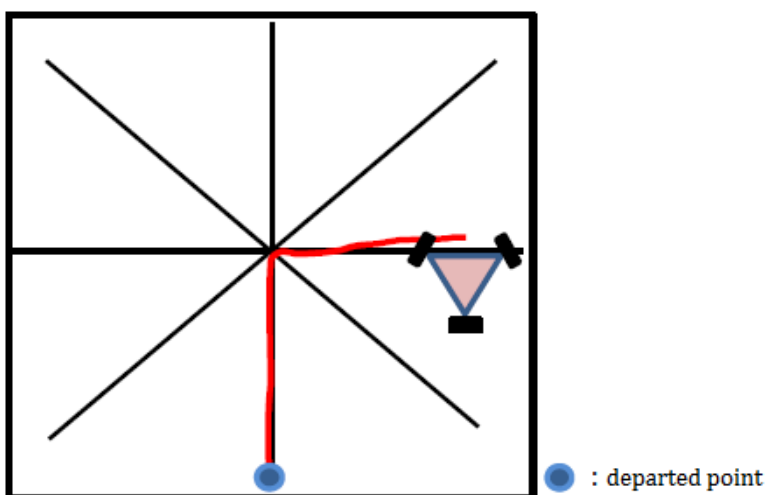

Fig. 14. The trajectory 5

The trajectory of the test was obtained by observing the five robot movements, the velocity value was obtained between $0.256 \mathrm{~m} / \mathrm{s}$ to $1.403 \mathrm{~m} / \mathrm{s}$ with an orientation error of $2.84 \%$.

\section{CONCLUSION}

With omnidirectional mobile robots that are capable of moving in both directions, the holonomic approach can be used. The Holonomic Motion System is a system which, without understanding the mechanical structure, represents the number of degrees of freedom. To determine the movement of the robot into the real trajectory in the area, based on the test results obtained by applying holonomic and inverse kinematics. In forward, backward, sideways, and diagonal movements, mobile robots are introduced. It is known that the movement of robot has a velocity of $0.256 \mathrm{~m} / \mathrm{s}$ to $1.403 \mathrm{~m} / \mathrm{s}$ and there is an orientation error of $2.84 \%$ based on the data measurement. An algorithm that is able to detect objects around the robot to prevent collisions can be implemented for further development. 


\section{REFERENCES}

[1] Yunardi, Riky Tri, Arief Muchadin, Kurnia Latifa Priyanti, and Deny Arifianto. "Inverse Kinematics and PID Controller Implementation of Hexapod Robot for Wall Follower Navigation." INAJEEE: Indonesian Journal of Electrical and Electronics Engineering 2, no. 2 (2019): 23 28.

[2] Utama, Khasia Vidra, Rifqi Amalya Fatekha, Senanjung Prayoga, Daniel S. Pamungkas, and Rizky Pratama Hudhajanto. "Positioning and Maneuver of an Omnidirectional Robot Soccer." In 2018 International Conference on Applied Engineering (ICAE), pp. 1-5. IEEE, 2018.

[3] Widodo, Faiz Anhar, and Kusprasapta Mutijarsa. "Design and implementation of movement, dribbler and kicker for wheeled soccer robot." In 2017 International Conference on Information Technology Systems and Innovation (ICITSI), pp. 200-205. IEEE, 2017.

[4] Latif, Abdul, K. Shankar, and Phong Thanh Nguyen. "Legged Fire Fighter Robot Movement Using PID." Journal of Robotics and Control (JRC) 1, no. 1 (2019): 15-19.

[5] He, Hongsheng, Shuzhi Sam Ge, and Guofeng Tong. "Task-Based Flocking Algorithm for Mobile Robot Cooperation." In FIRA RoboWorld Congress, pp. 310-321. Springer, Berlin, Heidelberg, 2009.

[6] Mülling, Katharina, Jens Kober, Oliver Kroemer, and Jan Peters. "Learning to select and generalize striking movements in robot table tennis." The International Journal of Robotics Research 32, no. 3 (2013): 263-279.

[7] Kim, Hongjun, and Byung Kook Kim. "Minimum-energy trajectory planning and control on a straight line with rotation for three-wheeled omni-directional mobile robots." In 2012 IEEE/RSJ International Conference on Intelligent Robots and Systems, pp. 3119-3124. IEEE, 2012.

[8] Kanjanawanishkul, Kiattisin. "Omnidirectional wheeled mobile robots: wheel types and practical applications." International Journal of Advanced Mechatronic Systems 6, no. 6 (2015): 289-302.

[9] Vejlupek, J., and V. Lamberský. "Multi-purpose mobile robot platform development." In Mechatronics, pp. 463-470. Springer, Berlin, Heidelberg, 2011.

[10] Ye, Changlong, and Shugen Ma. "Development of an omnidirectional mobile platform." In 2009 International Conference on Mechatronics and Automation, pp. 1111-1115. IEEE, 2009.

[11] Moreno, Javier, Eduard Clotet, Ruben Lupiañez, Marcel Tresanchez, Dani Martínez, Tomàs Pallejà, Jordi Casanovas, and Jordi Palacín. "Design, implementation and validation of the three-wheel holonomic motion system of the assistant personal robot (APR)." Sensors 16, no. 10 (2016): 1658.

[12] Deepak, B. B. V. L., and Dayal R. Parhi. "Kinematic analysis of wheeled mobile robot." Automation \& Systems Engineering 5, no. 2 (2011): 96-111.

[13] Bräunl, Thomas. Embedded robotics: mobile robot design and applications with embedded systems. Springer Science \& Business Media, 2008.

[14] Yildiz, Ibrahim. "A low-cost and lightweight alternative to rehabilitation robots: omnidirectional interactive mobile robot for arm rehabilitation." Arabian Journal for Science and Engineering 43, no. 3 (2018): 1053-1059.

[15] Sai, K. V. S., and R. Sivaramakrishnan. "Design and fabrication of holonomic motion robot using DTMF control tones." In 2009 International Conference on Control, Automation, Communication and Energy Conservation, pp. 1-4. IEEE, 2009.
[16] Maggiore, Manfredi, and Luca Consolini. "Virtual holonomic constraints for Euler-Lagrange systems." IEEE Transactions on Automatic Control 58, no. 4 (2012): 1001-1008.

[17] Freidovich, Leonid B., Uwe Mettin, Anton S. Shiriaev, and Mark W. Spong. "A passive 2-DOF walker: Hunting for gaits using virtual holonomic constraints." IEEE Transactions on robotics 25 , no. 5 (2009): 1202-1208.

[18] Amouri, Lobna, Cyril Novales, Mohamed Jallouli, Gerard Poisson, and Nabil Derbel. "An effective fuzzy-DVZ controller for an omnidirectional mobile robot." In International Multi-Conference on Systems, Signals \& Devices, pp. 1-6. IEEE, 2012.

[19] Maulana, Eka, M. Aziz Muslim, and Veri Hendrayawan. "Inverse kinematic implementation of four-wheels mecanum drive mobile robot using stepper motors." In 2015 International Seminar on Intelligent Technology and Its Applications (ISITIA), pp. 51-56. IEEE, 2015.

[20] Myint, Cherry, and Nu Nu Win. "Position and velocity control for twowheel differential drive mobile robot." International Journal of Science Engineering and Technology Research (IJSETR) 5, no. 9 (2016): 28492855.

[21] Shijin, C. S., and K. Udayakumar. "Speed control of wheeled mobile robots using pid with dynamic and kinematic modelling." In 2017 International Conference on Innovations in Information, Embedded and Communication Systems (ICIIECS), pp. 1-7. IEEE, 2017.

[22] Maldeniya, M. K. S. H., R. C. Madurawe, L. B. H. T. Thilakasiri, T. M. S. Thennakoon, and R. M. T. P. Rajakaruna. "Remote controlled 4WD omni directional robot using mecanum wheels." Department of Mechatronics, Faculty of Engineering, South Asian Institute of Technology and Medicine (SAITM), Sri Lanka (2015).

[23] Nagatani, Keiji, Seiga Kiribayashi, Yoshito Okada, Satoshi Tadokoro, Takeshi Nishimura, Tomoaki Yoshida, Eiji Koyanagi, and Yasushi Hada. "Redesign of rescue mobile robot Quince." In 2011 IEEE International Symposium on Safety, Security, and Rescue Robotics, pp. 13-18. IEEE, 2011.

[24] Prasojo, Ipin, Phong Thanh Nguyen, and Nishith Shahu. "Design of Ultrasonic Sensor and Ultraviolet Sensor Implemented on a Fire Fighter Robot Using AT89S52." Journal of Robotics and Control (JRC) 1, no. 2 (2020): 55-58.

[25] Mahzan, N. N., A. M. Omar, L. Rimon, SZ Mohammad Noor, and M. Z. Rosselan. "Design and development of an arduino based data logger for photovoltaic monitoring system." Int. J. Simul. Syst. Sci. Technol 17, no. 41 (2017): 15-1.

[26] Prisma Megantoro, Danar Aulia Husnan, Mian Usman Sattar, Andino Maseleno and Omar Tanane. "Validation Method for Digital Flow Meter for Fuel Vendors." Journal of Robotics and Control (JRC) 1, no. 2 (2020): 44-48.

[27] Rubies, Elena, and Jordi Palacín. "Design and FDM/FFF Implementation of a Compact Omnidirectional Wheel for a Mobile Robot and Assessment of ABS and PLA Printing Materials." Robotics 9, no. 2 (2020): 43.

[28] Hou, Linfei, Liang Zhang, and Jongwon Kim. "Energy Modeling and Power Measurement for Three-Wheeled Omnidirectional Mobile Robots for Path Planning." Electronics 8, no. 8 (2019): 843.

[29] Soliman, MennaAllah, Ahmad Taher Azar, Mahmood Abdallah Saleh, and Hossam Hassan Ammar. "Path planning control for 3-omni fighting robot using PID and fuzzy logic controller." In International Conference on Advanced Machine Learning Technologies and Applications, pp. 442-452. Springer, Cham, 2019.

[30] Arvin, Farshad, Khairulmizam Samsudin, and M. Ali Nasseri. "Design of a differential-drive wheeled robot controller with pulse-width modulation." In 2009 Innovative Technologies in Intelligent Systems and Industrial Applications, pp. 143-147. IEEE, 2009. 